\title{
Twelve-Week Aerobic Training Decreases Chemerin Level and Improves Cardiometabolic Risk Factors in Overweight and Obese Men
}

\begin{abstract}
Abbas Saremi ${ }^{1}$, PhD; Nader Shavandi ${ }^{1}$, PhD; Mohammad Parastesh ${ }^{1}$, PhD Candidate; $^{2}$
Hassan Daneshmand ${ }^{2}$, MD

Authors' Affiliation:

1. Department of Sport Sciences, Arak University, Arak, IR Iran

2. Department of Medical Sciences, University of Arak, Arak, IR Iran

* Corresponding Author; Address: Department of Sport Sciences, University of Arak, Arak, IR Iran

E-mail: a-saremi@araku.ac.ir

Received: Apr 24, 2010

Accepted: Jun 15, 2010

Key Words: Exercise; Training; Obesity; Metabolic syndrome; Chemerin

Abstract

Purpose: The inflammatory state of adipose tissue in obese subjects may be the most important factor linking increased adipose tissue mass to insulin resistance. Chemerin is a newly discovered adipokine that plays an important role in macrophage infiltration into adipose tissue and may contribute to the development of inflammation and insulin resistance. We examined the effects of 12 weeks of aerobic training on serum chemerin levels in association with cardiovascular risk factors in overweight and obese males.

Methods: Twenty-one overweight and obese subjects $[44.3$ ( $\pm 4.1 \mathrm{yrs}$, body mass index $(B M I) \geq 25 \mathrm{~kg} / \mathrm{m}^{2}$ ) were assigned to exercise training (obese EX, $n=11$ ) and control (obese $C O N, n=10)$ groups. The obese EX group participated in 12 weeks of progressive aerobic training 5 days a week. Serum chemerin, insulin resistance, lipid profiles, blood pressure, and body composition were all measured before and after the training.

Results: After the aerobic training, waist circumference $(P=0.009)$, fat percent $(P=0.03)$, visceral fat $(P=0.03)$, subcutaneous fat $(P=0.01)$, fasting glucose $(P=0.01)$, insulin resistance $(P=0.03)$, triglyceride $(P=0.05)$, total cholesterol $(P=0.04)$, low-density lipoprotein cholesterol $(P=0.05)$ and systolic blood pressure $(P=0.04)$ of participates were significantly decreased. Concurrently, serum chemerin concentrations were significantly decreased after aerobic program $(P=0.02)$.

Conclusion: Aerobic training caused an improvement in cardiometabolic risk factors in obese subjects, and this improvement was accompanied by decreased chemerin levels.
\end{abstract}

\section{INTRODUCTION}

$\mathrm{O}_{a}^{\mathrm{b}}$ besity, particularly visceral adiposity, is accompanied by chronic low-grade inflammation, indicated by increased serum levels of inflammatory markers such as C-reactive protein (CRP), tumor necrosis factor- $\alpha$ (TNF- $\alpha$ ), and interleukin- 6 (IL-6) of obese patients ${ }^{[1,2]}$. Chronic inflammation is a wellknown risk factor of insulin resistance and metabolic syndrome $^{[3]}$. It is now generally considered that 
macrophages infiltrating into obese adipose tissue from circulation are a primary source of inflammation in obesity $^{[4]}$ and could be involved in pathophysiology of insulin resistance ${ }^{[5]}$. Cellular and molecular mechanisms responsible for this macrophage infiltration are not clear.

Recently, it has been demonstrated that chemerin may play an important role in macrophage infiltration into adipose tissue ${ }^{[6-8]}$. Chemerin is a novel adipokine that has been reported to serve as a chemoattractant for macrophages via direct interaction with its receptors (chemokine-like receptor-1) ${ }^{[6-8]}$. It has been reported that chemerin expression in adipose tissue is increased in obese mice ${ }^{[9]}$. In addition, circulating levels of chemerin are elevated in obese and diabetics subjects [10]. Furthermore, in vitro studies revealed that treatment with chemerin induces insulin resistance in human skeletal muscle cells at the levels of IRS1 (Insulin receptor substrate 1), protein kinases B (PKB), and Glycogen synthase kinase 3 (GSK3) phosphorrylation and glucose uptake ${ }^{[1]]}$. Several studies have investigated the association between metabolic syndrome indices and chemerin ${ }^{[10-12]}$. In these studies chemerin levels were positively correlated with body mass index, waist circumference, blood pressure, triglyceride (TG), low-density lipoprotein cholesterol (LDL-C) levels and insulin resistance, and were inversely correlated with adiponectin and high-density lipoprotein cholesterol (HDL-C) levels. Taken together, these findings suggest that chemerin play an important role in macrophage infiltration into adipose tissue and may contribute to development of inflammation and insulin resistance.

Physical inactivity is a well known risk factor of type2 diabetes mellitus development ${ }^{[13]}$ and aerobic training has been shown to reduce adiposity and insulin resistance in obese adults ${ }^{[14]}$. While we know a lot about the mechanisms by which adiposity leads to insulin resistance ${ }^{[15]}$ and how exercise increases insulin sensitivity ${ }^{[16,17]}$, it hasn't been yet reported about the exercise-induced changes in chemerin concentrations, which may provide a link between obesity and insulin resistance. Therefore, the aim of this study was to assess the effect of aerobic training on serum chemerin in relation to improvement of insulin sensitivity in overweight and obese men.

\section{METHODS AND SUBJECTS}

Twenty-one overweight and obese, middle-aged and sedentary men [age $44.3( \pm 4.1)$ yrs, body mass index $(\mathrm{BMI}) \geq 25 \mathrm{~kg} / \mathrm{m}^{2}$ ] were recruited to the present study. All participants underwent an initial eligibility screening in exercise physiology laboratory of Arak University. Eligible participants were randomized to exercise training (obese EX, $\mathrm{n}=11$ ) and control (obese $\mathrm{CON}, \mathrm{n}=10$ ) groups. Figure 1 presents the distribution of study participants. The obese EX group participated in 12-week aerobic training program, while the obese CON maintained their lifestyle as usual. All subjects had a stable weight for at least 3 months before inclusion. We excluded candidates who were smoker, had cardiovascular diseases or any other major illnesses, or were taking medications that could affect laboratory test results. The physical activity level of subjects was assessed during an interview. The ethics committee of university approved the experimental procedures and study protocols, which were fully explained to all subjects. A written consent form was signed by each subject after having read and understood details of the experiments.

Dietary assessment: The subjects were instructed to follow their usual eating habits during the course of the study. Dietary intakes were registered during the 12-wk intervention period using 3-d dietary records. The participants were provided with several practice sessions regarding a measuring cup, spoon and ruled paper until they learned how to estimate the sizes of food items consumed. Then food records were analyzed using the food processor II nutrition systemanalysis software Version 3.1 (Tehran University of Medical Sciences, Iran) to determine total daily caloric and macronutrient intake.

Aerobic training program: Aerobic training supervised by an exercise physiologist was performed 50-60 $\mathrm{min} / \mathrm{d}, 5 \mathrm{~d} / \mathrm{wk}$, for 12 weeks. At each training session, those in the exercise group performed warmup exercises lasting $10 \mathrm{~min}$, followed by a $15-50 \mathrm{~min}$ walking-running, and $10 \mathrm{~min}$ of relaxation exercises at the end of the exercise period. Exercise intensity progressed from $15-20$ min at $60-65 \%$ of $\mathrm{HR}_{\max }$ during the first week to $25-30 \mathrm{~min}$ at $60-70 \%$ of $\mathrm{HR}_{\max }$ by week $3,35-40$ min at $75-80 \%$ of $\mathrm{HR}_{\max }$ by week 7 and 


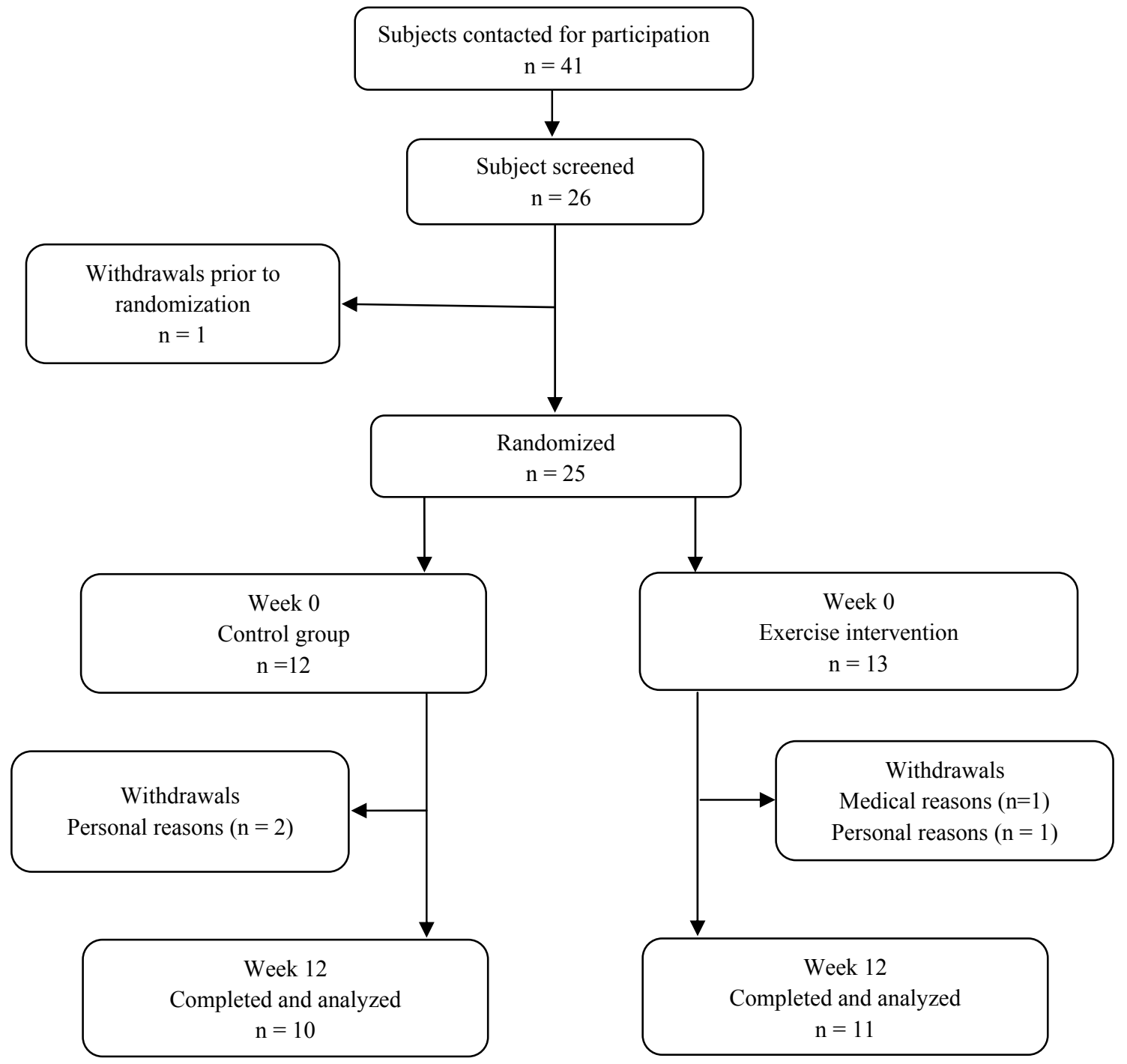

Fig. 1: Flow chart of subject participation.

45-50 min at $80-85 \%$ of $\mathrm{HR}_{\max }$ by week 12 . Target heart rates were monitored using telemetry. Aerobic training included treadmill walking/running. Peak oxygen consumption $\left(\mathrm{VO}_{2}\right.$ peak) was measured using a graded treadmill test and online computer-assisted open-circuit spirometry. Peak $\mathrm{VO}_{2}$ was attained when subjects achieved at least two of the following criteria: (a) a respiratory exchange ratio in excess of 1.0, (b) reaching $\geq 85 \%$ of age-predicted $\mathrm{HR}_{\max }$, or (c) achieving a rating of perceived exertion (Borg Scale) $\geq 17$.
Anthropometric and body composition measurements: Height $(\mathrm{m})$ and weight $(\mathrm{kg})$ were measured to calculate BMI as body weight $(\mathrm{kg}) /$ height $^{2}(\mathrm{~m})$. Waist circumference was measured at the narrowest point superior to the hip using a tape ruler. Whole body fat mass and percent body fat was assessed by bioelectric impedance analysis (Body composition analyzer, In Body 3.0, Korea). A singleslice computed tomography scan taken midway between L4 and L5 was performed using a Siemens Helicoidal Somatom Balance Scanner (Siemens, 
Erlangen, Germany) to measure visceral and subcutaneous adipose tissue areas as previously described $^{[18]}$.

Blood pressure and blood sample analysis: Blood pressure was measured twice, after a $10-$ min rest with a random zero mercury sphygmomanometer, and was averaged. All subjects reported for blood sampling in the morning after an overnight fast. Post- training blood samples $(10 \mathrm{ml})$ from subjects in the training group were obtained $48 \mathrm{~h}$ after their last exercise session. After collection, blood samples were serum-containing tubes were stored at $-70^{\circ} \mathrm{c}$ until analysis. Plasma total cholesterol, triglyceride, and HDL-C were measured by enzymatic colorimetric methods (Pars Azmun, Tehran, Iran). LDL-C was calculated using the formula of Friedewald. Plasma glucose was determined by the glucose oxidase method (Pars Azmun, Tehran, Iran). Plasma insulin was determined by chemiluminescent immunometric assay (Monobind Inc, USA). The homeostasis model assessment (HOMA), insulin resistance index, was calculated by using the formula:

fasting glucose $(\mathrm{mg} \mathrm{dl}-1) \times$ fasting insulin $(\mu \mathrm{U} \mathrm{ml} \mathrm{-1)}$

$$
\times 405-1 \text {. }
$$

Serum chemerin concentration was determined using a commercially available ELISA kit (Biovendor, Czech). The intra-assay coefficient of variation of total

cholesterol, TG, HDL-C, glucose, insulin, and chemerin were $1.1 \%, 1.6 \%, 1.9 \%, 1.28 \%, 5.3 \%$, and $5.1 \%$ respectively.

Statistical analysis: Data were presented as mean \pm SD. Normality assumption of the data was evaluated and was confirmed using one-sample KolmogorovSmirnov test in each group. Differences between normal weight and obese subjects were tested using the independent samples t-test. Changes in dependent variables resulting from the exercise intervention were assessed by two-way time-by-group repeated measures analysis of variance. The assumption of homogeneity of variances of groups was tested and confirmed by Levene test for equality of variances. Pearson's correlation was used to analyze various variables and changes in variables. $P<0.05$ was considered significant. The data were analyzed using SPSS (version 16) software.

\section{RESULTS}

The clinical and metabolic characteristics of participants before and after 12 weeks of training are presented in table 1. Obese EX group completed at least $94 \%$ of the exercise sessions. After 12 weeks of

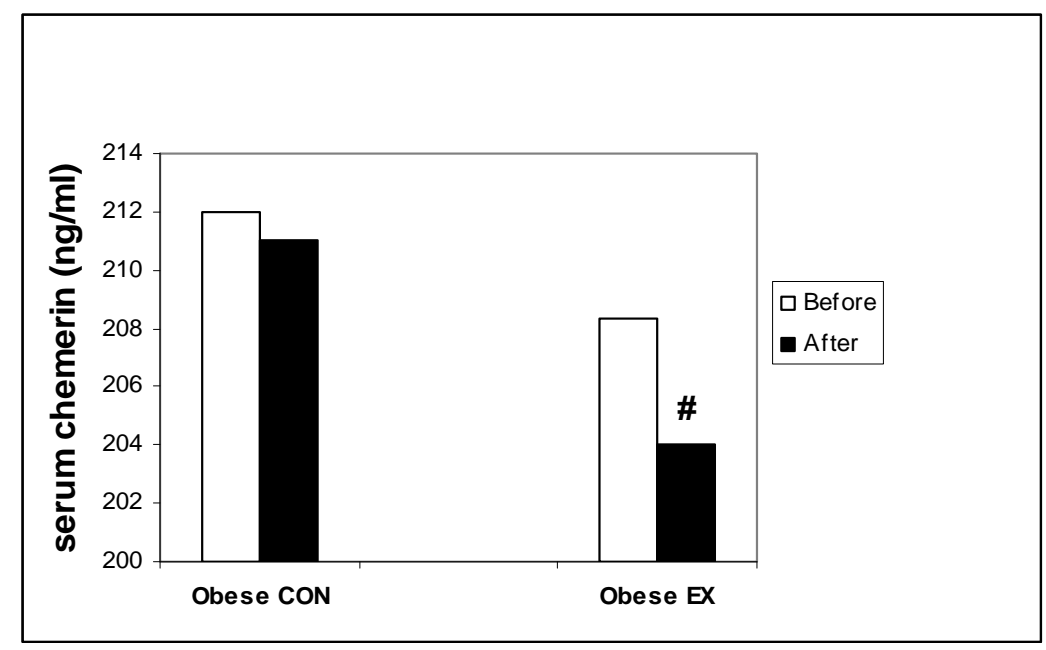

Fig. 2: Serum chemerin levels in normal weight and obese subjects before and after 12 weeks of aerobic training (Data are expressed as the mean $\pm \mathrm{SD}$ ).

${ }^{*}$ significant $(P<0.05)$ difference between before and after training. 
aerobic training, the obese EX group had weight loss $(P=0.02)$, which was accompanied with a significant decrease in BMI $(P=0.01)$, waist circumference (WC) ( $P=0.009)$, percent body fat $(P=0.03)$, subcutaneous fat $(P<0.01)$, visceral fat $(P=0.03)$, glucose $(P=0.01)$, HOMA-IR $(P=0.03)$, TG $(P=0.05)$, TC $(P=0.04)$, LDL$\mathrm{C}(P=0.05)$, and SBP $(P=0.04)$. Besides, $\mathrm{VO}_{2}$ peak increased significantly $(P=0.03) \quad$ (Table 1$)$ and chemerin concentrations were decreased significantly in obese EX group $(P=0.02)$ (Fig. 2). Furthermore, changes in chemerin concentrations after a 12 -week training period were correlated with alterations in visceral fat $(r=0.65, P=0.04)$, subcutaneous fat $(r=$
0.63, $P=0.05)$ HOMA-IR $(r=0.67, P=0.04)$, glucose $(r$ $=0.65, P=0.05)$, WC $(r=0.70, P=0.03)$, and $\mathrm{VO}_{2}$ peak $(r=-0.68, P=0.04)$. However, no significant changes were observed between pre- and post testing measurement of variables in the obese CON group $(P>0.05)$ (Table 1).

There were no significant differences in the amount of calories consumed $\left(\sim 35 \mathrm{kcal} \cdot \mathrm{kg}^{-1} \cdot \mathrm{d}^{-1}\right)$ or the percentage of calories obtained from carbohydrate $(\sim \% 58)$, protein $(\sim \% 20)$, and fat $(\sim \% 22)$ between groups during the 12-wk course of aerobic training $(P>0.05)$.

Table 1: Participant characteristics before and after 12 weeks of aerobic training

\begin{tabular}{|c|c|c|c|c|}
\hline \multirow{2}{*}{ Characteristics } & \multicolumn{2}{|c|}{ Obese CON } & \multicolumn{2}{|c|}{ Obese EX } \\
\hline & before & after & before & after \\
\hline \multicolumn{5}{|l|}{ Anthropometric } \\
\hline Weight (kg) & $96.31 \pm 6.65$ & $96.42 \pm 6.61$ & $97.81 \pm 5.22$ & $94.03 \pm 4.67 \#$ \\
\hline BMI $\left(\mathbf{k g} / \mathrm{cm}^{2}\right)$ & $29.54 \pm 1.82$ & $29.29 \pm 1.87$ & $29.11 \pm 1.69$ & $28.11 \pm 1.50 \#$ \\
\hline WC (cm) & $96.88 \pm 10.43$ & $97.55 \pm 10.45$ & $95.00 \pm 9.84$ & $91.88 \pm 7.75 \#$ \\
\hline Fat $(\%)$ & $35.13 \pm 2.31$ & $35.25 \pm 2.36$ & $35.69 \pm 2.74$ & $33.88 \pm 2.40 \#$ \\
\hline Visceral fat $\left(\mathrm{cm}^{2}\right)$ & $148.60 \pm 20.68$ & $149.00 \pm 20.69$ & $149.45 \pm 19.75$ & $146.63 \pm 19.06 \#$ \\
\hline Subcutaneous fat $\left(\mathrm{cm}^{2}\right)$ & $278.40 \pm 29.45$ & $280.10 \pm 30.31$ & $277 \pm 28.68$ & $272.10 \pm 24.91 \#$ \\
\hline \multicolumn{5}{|l|}{ Insulin resistance } \\
\hline Glucose $\left(\mathrm{mg} \mathrm{dl}^{-1}\right)$ & $98.55 \pm 12.09$ & $96.66 \pm 10.64$ & $99.66 \pm 12.74$ & $94.77 \pm 9.90 \#$ \\
\hline Insulin $\left(\mu \mathrm{U} \mathrm{ml}^{-1}\right)$ & $8.03 \pm 1.28$ & $8.17 \pm 1.04$ & $8.23 \pm 1.69$ & $8.15 \pm 1.47$ \\
\hline HOMA-IR & $1.98 \pm 0.53$ & $1.94 \pm 0.41$ & $2.06 \pm 0.67$ & $1.93 \pm 0.53 \#$ \\
\hline \multicolumn{5}{|l|}{ Lipid profile } \\
\hline TG (mg/dl) & $120.22 \pm 26.84$ & $122.55 \pm 27.87$ & $118.33 \pm 26.88$ & $116.11 \pm 27.94 \#$ \\
\hline $\mathrm{TC}(\mathrm{mg} / \mathrm{dl})$ & $201.77 \pm 22.80$ & $195.44 \pm 23.54$ & $198.44 \pm 21.88$ & $189.77 \pm 20.46 \#$ \\
\hline LDL-C (mg/dl) & $121.77 \pm 15.54$ & $123.00 \pm 16.94$ & $122.88 \pm 15.52$ & $118.22 \pm 17.80 \#$ \\
\hline HDL-C (mg/dl) & $48.33 \pm 9.23$ & $48.00 \pm 8.55$ & $47.77 \pm 9.79$ & $48.44 \pm 9.19$ \\
\hline \multicolumn{5}{|l|}{ Blood pressure } \\
\hline SBP (mm Hg) & $130.44 \pm 7.66$ & $129.77 \pm 7.41$ & $131.66 \pm 6.44$ & $129.33 \pm 7.05 \#$ \\
\hline DBP (mm Hg) & $87.77 \pm 7.69$ & $84.22 \pm 7.62$ & $87.22 \pm 8.59$ & $85.11 \pm 9.22$ \\
\hline $\operatorname{Vo2} 2_{\max }(1 / \min )$ & $2.17 \pm 0.45$ & $2.22 \pm 0.51$ & $2.13 \pm 0.40$ & $2.25 \pm 0.43 \#$ \\
\hline $\begin{array}{l}\text { ata are expressed as mean } \pm \mathrm{SD} \\
\text { significant }(P<0.05) \text { difference } \mathrm{b} \\
\text { 3MI: body mass index } / \mathrm{WC}=\text { wais } \\
\text { G: triglyceride/ TC: total cholest } \\
\text { BP: systolic blood pressure/ DBP }\end{array}$ & $\begin{array}{l}\text { e and after tra } \\
\text { nce/ HOMA-I } \\
\text { low-density } \\
\text { ood pressure/ }\end{array}$ & meostasis moc & sment for ins & $\begin{array}{l}\text { istance/ } \\
\text { protein cholesterol// }\end{array}$ \\
\hline
\end{tabular}




\section{DISCUSSION}

The main finding of this study was that 12 weeks of aerobic training at $60-85 \%$ of $\mathrm{HR}_{\text {max }}$ improved insulin resistance in overweight and obese subjects. The beneficial effect of increased physical activity on insulin sensitivity was accompanied by changes in body fat (i.e., visceral fat) and circulating chemerin levels.

Adipose tissue is an active organ which secretes many proteins known as adipokines that are metabolically important ${ }^{[19]}$. Some of these adipokines have important functions in incidence of insulin resistance and cardiovascular complications with obesity, especially central or visceral obesity ${ }^{[20]}$. Chemerin is a recently discovered adipokine produced and secreted primarily by adipose tissue ${ }^{[9,10]}$. In vitro studies have shown that chemerin induces insulin resistance at the levels of IRS1, Akt, and GSK3 phosphorylation and glucose uptake [11]. Taken together, these results suggested that chemerin could play a role in the association between abdominal obesity and increased metabolic risk.

Physical inactivity is related to most components of the metabolic syndrome ${ }^{[13]}$. Regular exercise results in reduction of risk factors of obesity, type 2 diabetes, and cardiovascular disease ${ }^{[14,21]}$. The current study demonstrated that the obese EX group that participated in the 12-week exercise intervention improved body fatness (i.e., body weight, BMI, percent body fat, visceral fat, subcutaneous fat), insulin resistance (i.e., fasting glucose, HOMA-IR), blood lipid profile (i.e., TG, TC, LDL), and resting blood pressure (i.e., SBP) in comparison with CON group. We also found that aerobic fitness was significantly increased in the exercise group compared with the control group. Our observations are consistent with previous reports and reinforce the positive effects of exercise training on these important risk factors of metabolic syndrome, type 2 diabetes, and cardiovascular disease ${ }^{[22-24]}$. In the present study, 12 weeks of aerobic training decreased insulin resistance by $4.7 \%$ when comparisons were made between pre- and post intervention levels, and this is in agreement with previous investigations ${ }^{[23,25]}$. We observed that reduction in subjects' abdominal obesity who ran for $\sim 60$ minutes, $5 \mathrm{~d} / \mathrm{wk}$ is consistent with Slentz et $\mathrm{al}^{[26]}$ who reported that overweight and no dieting subjects performed a $\sim 45$ min activity, $4 \mathrm{~d} / \mathrm{wk}$ had a marked reduction in abdominal (waist circumferences) obesity. These findings confirm those previously reported from similar well controlled trials ${ }^{[27]}$ and suggest that exercise without caloric restriction for $\sim 60$ minutes at $60-85 \%$ of $\mathrm{HR}_{\max }$ is associated with reductions in abdominal obesity. Indeed, these results confirm those of previous studies that found improvement in insulin sensitivity after aerobic training and abdominal obesity reduction in obese individuals ${ }^{[27]}$. Several mechanisms have been proposed to be responsible for the increases in insulin sensitivity after exercise training ${ }^{[16,17]}$. Changes in production of adipokines may play an important role in obesity-related cardiovascular complications and insulin resistance ${ }^{[19,28]}$ and it can help understand the beneficial effects of exercise.

The present study is the first to examine the influence of exercise training on chemerin serum levels. We observed a significant decrease in chemerin levels in concomitant with improvements in insulin resistance and obesity parameters after 12 weeks of aerobic training. This inverse relationship is in accordance with the theoretical negative role of chemerin in regulating insulin sensitivity and metabolic indices. It has been shown that chemerin concentrations increase with adiposity ${ }^{[9-12]}$. In agreement with these studies, our results showed that chemerin levels decreased significantly after body weight reduction (particularly visceral fat) in obese subjects. The decrease in chemerin concentrations indicates that changes in abdominal fat after 12 weeks of aerobic training may play an important role in the regulation of macrophage infiltration into adipose tissue and serum inflammatory markers (i.e., chemerin). Recently, surgery-induced weight loss in severely obese patients was demonstrated to reduce macrophage infiltration into adipose tissue ${ }^{[29]}$.

However, the present paper demonstrates that weight loss obtained through aerobic training induces a pronounced improvement in inflammation marker (chemerin) and metabolic syndrome indices. Taken together, our finding suggests that a relatively short course of training is sufficient to reduce chemerin levels, and probably, at least in part, induces early 
improvements in insulin resistance and other cardio metabolic risk factors after exercise related to the decrease in chemerin levels. Based on the critical important of inflammation in favoring cardiovascular and metabolic abnormalities in obesity, it could be suggested that obese subjects need to participate in aerobic training programs that lead to loss of body weight and fat reduction.

One limitation of this study was that we measured only one of the chemokines that are known to regulate inflammatory process. Clearly, other chemokines (e.g., monocyte chemoattractant protein-1) that we did not measure are also involved in the regulation of inflammation, and insulin resistance.

\section{CONCLUSION}

A 12-week aerobic training program decreased insulin resistance and abdominal obesity, and chemerin concentrations in overweight and obese subjects. These findings suggest that exercise-induced changes in chemerin levels may be associated with the beneficial effects of exercise. Further studies are needed to elucidate the mechanisms by which exercise affects chemerin and metabolic syndrome.

\section{ACKNOWLEDGMENTS}

This work was supported by Arak University Research Center (grant numbers: 87/13986). The authors thank the subjects who participated in this study and the laboratory assistants in the clinical laboratory center of Arak university hospital who assisted with data collection and analysis.

Conflict of interests: The authors declare that they have no relevant financial interests in this manuscript.

\section{REFERENCES}

1. Compher C, Badellino KO. Obesity and inflammation: lessons from bariatric surgery. J Parenter Enteral Nutr. 2008;32:645-7.

2. Schenk S, Saberi M, Olefsky JM. Insulin sensitivity: modulation by nutrients and inflammation. J Clin Invest. 2008;118:29923002 .

3. Xu H, Barnes GT, Yang Q, et al. Chronic inflammation in fat plays a crucial role in the development of obesity-related insulin resistance. J Clin Invest. 2003;112:1821-30.

4. Cancello R, Clément K. Is obesity an inflammatory illness? Role of low-grade inflammation and macrophage infiltration in human white adipose tissue. BJOG. 2006;113:1141-7.

5. Fernández-Veledo S, Nieto-Vazquez I, Vila-Bedmar R, et al. Molecular mechanisms involved in obesity-associated insulin resistance: therapeutical approach. Arch Physiol Biochem. 2009;115:227-39.

6. Goralski KB, McCarthy TC, Hanniman EA, et al. Chemerin, a novel adipokine that regulates adipogenesis and adipocyte metabolism. J Biol Chem. 2007;282:28175-88.

7. MacDougald OA, Burant CF. The rapidly expandingfamily of adipokines. Cell Metab. 2007;6:159-61.

8. Zabel BA, Ohyama T, Zuniga L, et al. Chemokine-like receptor 1 expression by macrophages in vivo: regulation by TGF-beta and TLR ligands. Exp Hematol. 2006;34:1106-14.

9. Roh SG, Song SH, Choi KC, et al. Chemerin--a new adipokine that modulates adipogenesis via its own receptor. Biochem Biophys Res Commun. 2007;362:1013-1018.

10. Wang LY, Wei L, Yu HY, et al. Relationship of serum chemerin to obesity and type 2 diabetes mellitus. Zhonghua Yi Xue Za Zhi. 2009;89:235-8.

11. Sell H, Laurencikiene J, Taube A, et al. Chemerin is a novel adipocyte-derived factor inducing insulin resistance in primary human skeletal muscle cell. Diabetes. 2009;58:2731-40. 
12. Bozaoglu K, Segal D, Shields KA, et al. Chemerin is associated with metabolic syndrome phenotypes in a Mexican-American population. J Clin Endocrinol Metab. 2009;94:3085-8.

13. Venables MC, Jeukendrup AE. Physical inactivity and obesity: links with insulin resistance and type 2 diabetes mellitus. Diabetes Metab Res Rev. 2009;25:S18-23.

14. O'Leary VB, Marchetti CM, Krishnan RK, et al. Exercise-induced reversal of insulin resistance in obese elderly is associated with reduced visceral fat. J Appl Physiol. 2006;100:1584-9.

15. Goossens GH. The role of adipose tissue dysfunction in the pathogenesis of obesity-related insulin resistance. Physiol Behav. 2008;94:206-18.

16. Ostergard T, Jessen N, Schmitz O, et al. The effect of exercise, training, and inactivity on insulin sensitivity in diabetics and their relatives: what is new? Appl Physiol Nutr Metab. 2007;32:541-8.

17. You T, Nicklas BJ. Effects of exercise on adipokines and the metabolic syndrome. Curr Diab Rep. 2008;8:7-11.

18. Ibanez J, Izquierdo M, Argüelles I, et al. Twice-weekly progressive resistance training decreases abdominal fat and improves insulin sensitivity in older men with type 2 diabetes. Diabetes Care. 2005;28:662-7.

19. Antuna-Puente B, Feve B, Fellahi S, et al. Adipokines: the link between insulin resistance and obesity. Diabetes \& Metabolism. 2008;34:2-11.

20. Rabe K, Lehrke M, Parhofer KG, Broedl UC. Adipokines and insulin resistance. Mol Med. 2008;14:741-51.

21. Lazarevic G, Antic S, Cvetkovic T, et al. Effects of regular exercise on cardiovascular risk factors profile and oxidative stress in obese type 2 diabetic patients in regard to SCORE risk. Acta Cardiol. 2008;63:485-91.

22. Chang LC, Huang KC, Wu YW, et al. The clinical implications of blood adiponectin in cardiometabolic disorders. $J$ Formos Med Assoc. 2009;108:353-66.

23. Davidson LE, Hudson R, Kilpatrick K, et al. Effects of exercise modality on insulin resistance and functional limitation in older adults: a randomized controlled trial. Arch Intern Med. 2009;69:122-31.

24. Prasad DS, Das BC. Physical inactivity: a cardiovascular risk factor. Indian J Med Sci. 2009;63:33-42.

25. Kamijo T, Murakami M. Regular physical exercise improves physical motor functions and biochemical markers in middle-age and elderly women. J Phys Act Health. 2009;6:55-62.

26. Slentz CA, Duscha BD, Johnson JL, et al. Effects of the amount of exercise on body weight, body composition, and measures of central obesity: STRRIDE-a randomized controlled study. Arch Intern Med. 2004;164:31-9.

27. Ross R, Janssen I, Dawson J, et al. Exercise-induced reduction in obesity and insulin resistance in women: a randomized controlled trial. Obese Res. 2004;12:789-98.

28. Hajer GR, Van Haeften TW, Visseren FL. Adipose tissue dysfunction in obesity, diabetes, and vascular diseases. Eur Heart J. 2008;29:2959-71.

29. Cancello R, Henegar C, Viguerie N, et al. Reduction of macrophage infiltration and chemoattractant gene expression changes in white adipose tissue of morbidly obese subjects after surgery-induced weight loss. Diabetes. 2005;54:2277-86. 